\title{
Controle glicêmico e suas evidências para prevenção de infecção de sítio cirúrgico em cirurgias de coluna
}

\author{
Glycemic control and its evidence for prevention of surgical site infection in spine surgeries \\ Control glucemico y sus evidencias para la prevención de infección de sitio quirurjico em \\ cirurgías de columna
}

Maria Fernanda Lopes ${ }^{1}$, Tamiris Mayra Martins de Souza ${ }^{1}$, Gislaine Cristhina Bellusse ${ }^{1 *}$.

\begin{abstract}
RESUMO
Objetivo: Analisar as evidências disponíveis na literatura sobre o controle glicêmico como medida de prevenção para a infecção de sítio cirúrgico (ISC) em cirurgias de coluna. Métodos: Revisão integrativa desenvolvida com a seleção de artigos primários nas bases de dados Medical Literature Analysisand Retrieval System online (MEDLINE), Biblioteca Virtual em Saúde (BVS) e no Portal de Periódicos da Coordenação de Aperfeiçoamento de Pessoal de Nível Superior (CAPES) utilizando-se os descritores: Glycemiccontrol, woundinfection, spine, spinesurgery, surgical site infection e hemoglobinaA1c. Resultados: Os resultados evidenciaram a hiperglicemia como fator de risco independente para ISC em pacientes submetidos a cirurgia de coluna. O nível de hemoglobina glicada foi associado à infecção de ISC sendo que o risco se torna maior à medida em que há aumento do nível de hemoglobina glicada. O Diabetes Mellitus foi apontado como preditor independente significativo para ISC. Considerações finais: A hiperglicemia perioperatória, independentemente da condição de Diabetes Mellitus, está relacionada a ocorrência de ISC. Observou-se a falta de parâmetros quanto o nível glicêmico ideal o que aponta a necessidade de novos estudos capazes de nortear essa lacuna.
\end{abstract}

Palavras-chave: Infecção da ferida operatória, Índice glicêmico, Coluna vertebral.

\begin{abstract}
Objective: To analyze the evidence available in the literature on glycemic control as a preventive measure for surgical site infection (SSI) in spine surgeries. Methods: Integrative review developed with the selection of primary articles in the databases of Medical Literature Analysis and Retrieval System online (MEDLINE), Virtual Health Library (VHL) and in the Periodical Portal of the Coordination for the Improvement of Higher Level Personnel (CAPES) using the descriptors: Glycemic control, wound infection, spine, spine surgery, surgical site infection and hemoglobin A1c. Results: Results showed hyperglycemia as an independent risk factor for SSI in patients undergoing spine surgery. The A1c level has been associated with SSI infection, and the risk becomes higher as the A1c level increases. Diabetes Mellitus has been shown to be a significant independent predictor for SSI. Final considerations: Perioperative hyperglycemia, regardless of the condition of Diabetes Mellitus, is related to the occurrence of SSI. The lack of parameters as to the ideal glycemic level was observed, which points to the necessity of new studies capable of guiding this gap.
\end{abstract}

Keywords: Operative wound infection, Glycemic index, Spine.

\section{RESUMEN}

Objetivo: Analizar las evidencias disponibles en la literatura sobre el control glucémico como medida de prevención para la infección de sitio quirúrgico (ISC) em cirugías de columna. Métodos: Revisión integradora desenvuelta con la selección de artículos primarios de las bases de datos Medical Literature Analysis and Retrieval System online (MEDLINE), Biblioteca Virtual de la Salud (BVS) y e nel Portal de Periódicos de la Coordinación de Mejora de Personas de Nivel Superior (CAPES) utilizando los descriptores: glucemic control,

1Universidade de Franca (Unifran), Franca - SP. *E-mail: gislaine.bellusse@unifran.edu.br 
woundinfection, spine, spinesurgery, surgical site infection y hemoglobina A1c. Resultados: Los resultados evidencian la hiperglucemia como factor de riesgoin de pendiente para ISC en pacientes sometidos a cirugía de columna. El nivel de hemoglobina glucosila da fue asociado a la infección de ISC siendo el riesgo mayora medida que aumenta dicho nivel de hemoglobina glucosilada. La Diabetes Mellitus fue determinada como potenciador de la ISC. Consideraciones finales: La hiperglucemia perioperatoria, independientemente de la condición de la Diabetes Mellitus, está relacionada com la aparición de ISC. Se observa falta de parâmetros encuanto al nível glucémico ideal lo que indicala necesidad de nuevos estúdios capaces de profundizare nesa laguna.

Palabras clave: Infección de herida quirúrgica, Índice glucémico, Columna vertebral.

\section{INTRODUÇÃO}

O controle de infecção hospitalar sofreu evolução no decorrer dos anos e assumiu papel relevante por atuar em todos os serviços de saúde que desenvolvem procedimentos capazes de interferir no surgimento das infecções. A infecção hospitalar $(\mathrm{IH})$ ultrapassa as dimensões evidentes relacionando-se de forma multifacetada às ações de saúde e deve ser considerada, não apenas como um fenômeno biológico, mas também como um evento histórico e social, o qual demanda pesquisas científicas na busca de evidências quanto às medidas de prevenção e controle (PEREIRA MS, et al., 2005).

O Ministério da Saúde, na portaria ํo 2616 de 1998, define IH, também considerada Infecção Relacionada à Assistência à Saúde (IRAS), como aquela cujas manifestações ocorrem após setenta e duas horas de internação, ou, antes desse período, quando relacionados aos procedimentos realizados dentro do hospital (BRASIL, 1998). Quando ela ocorre em regiões manipuladas ou incisionadas em um procedimento cirúrgico, denomina-se infecção de sítio cirúrgico (ISC), desde que se apresente em até trinta dias após intervenção realizada, ou em até noventa dias em pacientes que necessitaram de implantação de próteses (AGÊNCIA NACIONAL DE VIGILÂNCIA SANITÁRIA, 2019).

O avanço tecnológico nos últimos anos foi responsável, entre outras questões, pelo aumento do número de cirurgias, o que se torna benéfico aos pacientes, no entanto, esses procedimentos cirúrgicos complexos e rotineiros deixam o ambiente cirúrgico, na maioria das vezes, inseguro (GOMES JAP, et al., 2016). Segundo Bezerra WR, et al. (2015), os episódios mais comuns evolvendo procedimentos cirúrgicos foram dor aguda no pós-operatório, retirada precipitada de dispositivos tubulares, reações adversas a medicamentos e, principalmente a IH.

As ISC têm importância no cenário hospitalar tanto a nível mundial quanto nacional, com incidência variável a depender das ações preventivas de cada serviço de saúde. Mundialmente, é a causa mais frequente de IRAS sendo que, no Brasil, a prevalência varia em torno de 14-16\% das infecções hospitalares (FUSCO SFB, et al., 2016). Esse destaque deve-se ao prejuízo socioeconômico, causando aumento da mortalidade, atraso das atividades laborais com impacto financeiro, instabilidade nas relações familiares além do déficit econômico aos serviços de saúde devido ao aumento dos gastos com hospitalização (CARVALHO RLR, et al.,2017).

Fatores intrínsecos e extrínsecos estão envolvidos no risco de ISC. Entre os fatores intrínsecos destacamse à desnutrição, idade extrema, neoplasias, obesidades, entre outros e, quanto aos fatores extrínsecos podemos citar tempo de internação prolongado, antibiótico profilaxia, processos de esterilização dos materiais, tempo de degermação cirúrgica das mãos e antebraços, técnica cirúrgica, entre outros.

Apesar de alguns fatores não serem passíveis de modificação, como, por exemplo, a idade do paciente, outros, por sua vez, podem e devem ser minimizados e até mesmo evitados, a fim de se obter sucesso nos resultados e, o mais importante, sem causar danos ao paciente (SOBECC, 2017). Dentre os fatores de risco relacionado à ISC, ressalta-se a hiperglicemia, caracterizada por alteração metabólica que tem efeito imunossupressor, pois está envolvida na função anormal dos leucócitos considerados importantes no papel de defesa do hospedeiro durante a primeira fase da cicatrização, denominada fase inflamatória, que é iniciada após uma lesão tecidual, decorrente do procedimento cirúrgico, envolvendo alterações vasculares, 
caracterizadas por aumento do fluxo sanguíneo através da dilatação das arteríolas e aumento da permeabilidade vascular e eventos celulares envolvendo os leucócitos.

Esses são recrutados por meio de uma sequência de eventos celulares que envolvem marginação e rolamento, adesão ao endotélio, transmigração por diapedese e quimiotaxia; em sequência, haverá a ativação dos leucócitos que promoverá a fagocitose, destruição intracelular de microrganismos e células mortas e produção de mediadores que amplificarão a resposta inflamatória. Ressalta-se que a hiperglicemia afetaa função dos leucócitos ao reduzir a quimiotaxia, aderência, fagocitose de microrganismos e função bactericida, consequentemente reduzindo a eliminação de microrganismos e aumentando o risco de infecção (KUMAR V, et al., 2016; TOWNSENDJRCM, et al., 2015).

Diante do aumento da população com Diabetes Mellitus (DM) ou que apresenta estado hiperglicêmico, o conhecimento da influência dos níveis glicêmicos no período perioperatório e sua relação com a ISC, tornouse imprescindível (DAVIS MC, et al., 2012). Vários fatores como o DM, a resposta endócrino-metabólica ("hiperglicemia estresse induzida"), efeitos da anestesia, bem como doenças críticas, podem ser responsáveis pela hiperglicemia perioperatória. As respostas endócrino-metabólicas podem ter início no pré-operatório devido a ansiedade e medo da anestesia, tal qual do procedimento cirúrgico (GUEDES AA, 2010).

Apesar de muito comum, a hiperglicemia ainda é considerada um grave problema e está relacionada a desfechos negativos como, por exemplo, ISC, mortalidade, dentre outros. Tanto os pacientes com DM como os que não possuem essa condição, podem apresentar hiperglicemia durante o período de internação hospitalar e, para tanto, torna-se necessário a implementação de medidas, no tocante ao manejo multidisciplinar no perioperatório, que visem a prevenção de ISC (THOMPSON M B, et al., 2016).

Diante dos dados expostos, justifica-se a condução deste estudo no intuito de contribuir para o conhecimento científico dos profissionais de saúde no tocante ao controle glicêmico no período perioperatório. Nessa perspectiva, o presente estudo teve como objetivo analisar as evidências disponíveis na literatura sobre o controle glicêmico como medida de prevenção para a ISC em cirurgias de coluna.

\section{MÉTODOS}

Trata-se de uma Revisão Integrativa (RI), método que possibilita a análise de todas as informações disponíveis na literatura sobre um determinado tema e sintetizá-las de modo que permita formular conclusões gerais, apontar os déficits encontrados ou propor novas medidas que auxiliem no manejo do assunto (MENDES KDS, et al., 2008; ERCOLE FF, et al., 2014).

Dentre as etapas definidas para esse tipo de estudo, utilizaram-se as seguintes: elaboração do tema, pesquisa dos descritores, estabelecimento de critérios para inclusão e exclusão de estudos, obtenção das informações a serem extraídas dos estudos, estudo dos artigos inclusos e por fim, análise dos resultados.

A estratégia Patientor Problem, Intervention, Controlor Comparasion, Outcomes (PICO) foi utilizada neste estudo para elaboração da questão de pesquisa (Quadro 1) (SANTOS CMC, et al., 2007).

Quadro 1 - Emprego da estratégia PICO - Franca, Brasil, 2020.

\begin{tabular}{|l|l|}
\hline $\mathrm{P}$ (paciente) & Paciente submetido a cirurgia de coluna \\
\hline $\mathrm{I}$ (intervenção) & Controle glicêmico no período perioperatório \\
\hline $\mathrm{C}$ (controle) & Não há \\
\hline $\mathrm{O}$ (resultado) & Ocorrência de ISC \\
\hline
\end{tabular}

Fonte: Lopes MF, et al., 2020.

A questão de pesquisa que norteou a condução desta revisão foi: "qual a relação entre o controle glicêmico no período perioperatório e a ocorrência de ISC em pacientes adultos submetidos a cirurgias de coluna?"

A busca dos descritores foi realizada no mês de maio de 2020 e ocorreu nas bases de dados Medical Literature Analysis and Retrieval System online (MEDLINE), Biblioteca Virtual em Saúde (BVS) e no Portal de Periódicos da Coordenação de Aperfeiçoamento de Pessoal de Nível Superior (CAPES). 
Foram selecionados e pesquisados os descritores controlados de acordo com o objetivo do trabalho, sendo os escolhidos: Glycemic control, wound infection, spine, spinesurgery, surgical site infection e hemoglobina A1c.

Os critérios de inclusão da presente pesquisa foram: estudos relacionados ao controle glicêmico como estratégia para prevenção de ISC no período perioperatório; estudos publicados nos idiomas português, inglês e espanhol entre o período de 2013 a 2020 e estudos com população de adultos maiores de 18 anos. Os critérios de exclusão delimitados foram estudos de revisão.

Após essa etapa, a síntese dos dados incluídos nesta RI foi realizada de forma descritiva e as seguintes informações foram inseridas: tipo de estudo, tamanho da amostra, objetivo do estudo, intervenção implementada e principais resultados (Figura 1).

Figura 1 - Fluxograma para seleção dos artigos incluídos na revisão - Franca, Brasil, 2020.

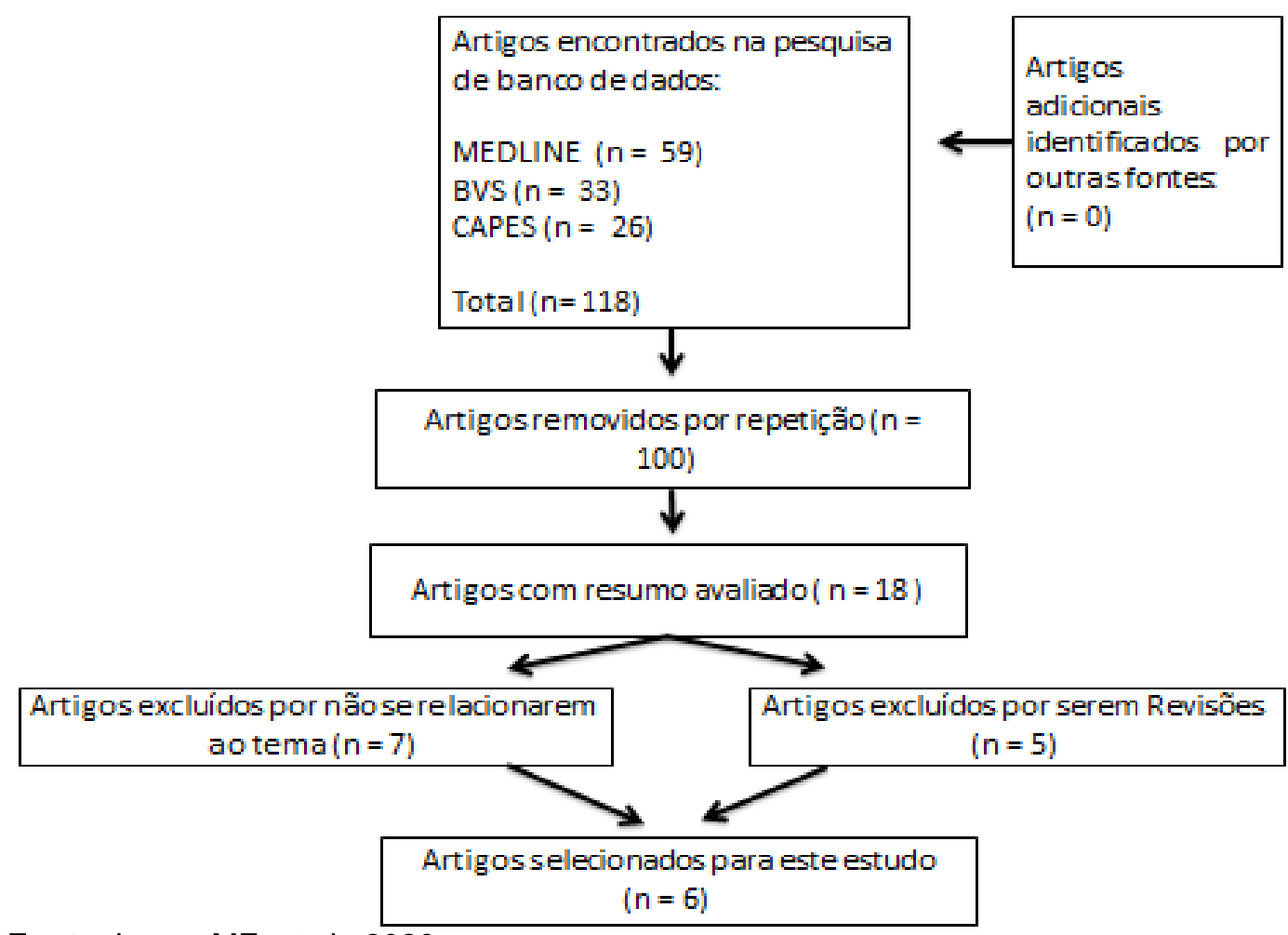

Fonte: Lopes MF, et al., 2020.

Nesta avaliação foram encontrados 118 artigos, sendo 59 deles no MEDLINE (PUBMED), 33 na BVS e 26 no Portal CAPES. Do total encontrado, observou-se repetição em 100 deles os quais foram excluídos deste estudo. Dos 18 artigos selecionados, 7 foram excluídos por não apresentarem títulos e resumos relacionados ao objetivo deste trabalho e 5 por se tratar de estudos de revisão, resultando em 6 artigos que foram minuciosamente analisados e incluídos na presente RI. Ressalta-se que os processos de busca e seleção dos estudos teóricos foram realizados por dois revisores de forma independente e as divergências foram discutidas até o alcance de concordância entre as partes envolvidas.

\section{RESULTADOS E DISCUSSÃO}

Dos 6 artigos incluídos nesta RI, verificou-se que todos foram publicados no idioma inglês, nos referidos periódicos: Revista Neuro Surgery, The Spine Journal, European Spine Journal, Medicine, Journal of Orthopaedic Science e Korean Journal of Neurotrauma. Em relação aos países de origem das publicações, houve predomínio dos Estados Unidos. A síntese dos estudos primários incluídos nesta RI, bem como os principais resultados e conclusões, está apresentada no Quadro 2. 
Quadro 2 - Síntese dos estudos primários incluídos na revisão integrativa.

\begin{tabular}{|c|c|c|c|c|}
\hline Estudo & Tipo de estudo & Amostra & Objetivo/ Intervenção & Resultado/Conclusão \\
\hline $\begin{array}{l}\text { Pennington Z, } \\
\text { et al. (2019). }\end{array}$ & Coorte Retrospectivo & $n=117$ & $\begin{array}{l}\text { Investigar hiperglicemia perioperatória } \\
\text { como fator de risco independente para } \\
\text { ISC. }\end{array}$ & $\begin{array}{c}\text { Preditores importantes de infecção: IMC; DM; variação } \\
\text { máxima no nível glicêmico; pico glicêmico no pós-operatório; } \\
\text { aumento da variabilidade dos picos glicêmicos no } \\
\text { perioperatório. A hiperglicemia perioperatória apresentou-se } \\
\text { como fator de risco independente para ISC em pacientes } \\
\text { submetidos a cirurgia de coluna (degenerativa). }\end{array}$ \\
\hline $\begin{array}{l}\text { Cancienne } \\
\text { JM, et al. } \\
\text { (2017 a). }\end{array}$ & $\begin{array}{l}\text { Caso Controle } \\
\text { Retrospectivo }\end{array}$ & $n=5.194$ & $\begin{array}{c}\text { Avaliar a associação do controle } \\
\text { glicêmico perioperatório por meio da } \\
\text { hemoglobina glicada }(\mathrm{HbA1c}) \text { em } \\
\text { pacientes com diabetes submetidos à } \\
\text { descompressão lombar com a incidência } \\
\text { de ISC profunda e calcular um limite de } \\
\text { HbA1c que possa relacionar-se com ISC. }\end{array}$ & $\begin{array}{c}\text { Taxa de infecção variou entre } 0,5-3,5 \% \text { em pacientes com } \\
\text { HbA1c }>11 \mathrm{mg} / \mathrm{dL} \text { e o ponto de inflexão da curva dos } \\
\text { pacientes analisados correspondeu a um nível de } \mathrm{HbA} 1 \mathrm{c} \text { de } \\
7,5 \mathrm{mg} / \mathrm{dL} \text {. O risco de ISC aumenta progressivamente quando } \\
\text { o nível de HbA1c ultrapassa } 7,5 \mathrm{mg} / \mathrm{dL} \text {. }\end{array}$ \\
\hline $\begin{array}{l}\text { Satake K, et } \\
\text { al. (2013). }\end{array}$ & Coorte Retrospectivo & $n=566$ & $\begin{array}{l}\text { Determinar fatores predisponentes para } \\
\text { ISC em pacientes com DM submetidos a } \\
\text { cirurgia de coluna vertebral. }\end{array}$ & $\begin{array}{l}\text { Fatores que aumentam o risco de ISC: DM, proteinúria pré- } \\
\text { operatória; tempo de operação e perda sanguínea estimada. } \\
\text { Pacientes com DM tinham risco aumentado em nove vezes e, } \\
\text { relação aos não diabéticos, porém não houve diferença } \\
\text { quanto ao controle glicêmico pré-operatório entre os dois } \\
\text { grupos. }\end{array}$ \\
\hline $\begin{array}{l}\text { Peng W, et } \\
\text { al. (2019). }\end{array}$ & Coorte Retrospectivo & $n=523$ & $\begin{array}{l}\text { Analisar fatores de risco para infecção } \\
\text { do sítio cirúrgico em pacientes } \\
\text { submetidos a cirurgia de coluna lombar. }\end{array}$ & $\begin{array}{l}\text { Condições que aumentam o risco de ISC: tabagismo, HbA1c } \\
\text { pré-operatória }>7,5 \mathrm{mg} / \mathrm{dL} \text {, albumina pós-operatória, tempo de } \\
\text { cirurgia, perda sanguínea, glicemia de jejum no pós- } \\
\text { operatório, espessura subcutânea local. O controle desses } \\
\text { fatores reduz, significativamente, o risco de ISC. }\end{array}$ \\
\hline $\begin{array}{l}\text { Hikata T, et } \\
\text { al. (2013). }\end{array}$ & Retrospectivo & $n=345$ & $\begin{array}{c}\text { Avaliar fatores de risco perioperatórios } \\
\text { para ISC após artrodese de coluna } \\
\text { vertebral. }\end{array}$ & $\begin{array}{c}\text { Pacientes com DM apresentaram maior taxa de ISC em } \\
\text { comparação aos não-DM; assim como foi maior em pacientes } \\
\text { com nível de } \mathrm{HbA} 1 \mathrm{c} \geq 7 \mathrm{mg} / \mathrm{dL} \text {. O controle de HbA1c menor que } \\
7 \mathrm{mg} / \mathrm{dL} \text { diminui a ocorrência de ISC. }\end{array}$ \\
\hline $\begin{array}{l}\text { Hwang JU, et } \\
\text { al. (2019). }\end{array}$ & Coorte Retrospectivo & $n=92$ & $\begin{array}{l}\text { Investigar a relação entre o nível de } \\
\text { hemoglobina A1c }(\mathrm{HbA} 1 \mathrm{c}) \text { e a incidência } \\
\text { de ISC após cirurgia de coluna lombar. }\end{array}$ & $\begin{array}{c}\text { O nível de HbA1c no pré-operatório foi maior nos pacientes } \\
\text { com ISC }(6,8 \%) \text { do que nos pacientes não infectados. A } \\
\text { análise mostrou que, o risco de ISC aumenta com um nível de } \\
\mathrm{HbA} 1 \mathrm{c} \geq 6,9 \% \text {. Assim, o nível de } \mathrm{HbA} 1 \mathrm{c}<6,9 \% \text {, diminui a } \\
\text { ocorrência de ISC. }\end{array}$ \\
\hline
\end{tabular}

Fonte: Lopes MF, et al., 2020.

REAS/EJCH | Vol.12(10) | e4606 | DOI: https://doi.org/10.25248/reas.e4606.2020 Página 5 de 9 
Os estudos incluídos abordaram cirurgia de coluna vertebral, em pacientes com ou sem diabetes mellitus (DM) e as amostras variaram de 92 a 5.194 pacientes (SATAKE K, et al., 2013; HIKATA T, et al., 2013; CANCIENNE JM, et al., 2017 a; PENNINGTON Z, et al., 2019; PENG W, et al., 2019; HWANG JU, et al., 2019). O DM é definido como um distúrbio metabólico caracterizado por hiperglicemia mantida que ocorre devido à insuficiência da liberação da insulina pelo pâncreas, da sua ação alterada ou de ambos os acontecimentos concomitantemente, aumentando a morbidade nos pacientes acometidos (SOCIEDADE BRASILEIRA DE DIABETES, 2019).

Independentemente da condição de DM, a hiperglicemia perioperatória torna-se responsável por resultados prejudiciais aos pacientes uma vez que podem ocasionar piores prognósticos devido à falta de adaptação do organismo ao estado de hiperglicemia aguda e ao processo inflamatório e oxidativo associados (FRISCH A, et al, 2010). Diante disso, os cuidados de saúde prestados podem ser negligenciados para os pacientes que apresentam a condição de $\mathrm{DM}$, mas, no entanto, não possuem diagnóstico prévio estabelecido (PALERMO NE, et al., 2016).

Dois estudos primários apresentaram como principais resultados que o nível de $\mathrm{HbA} 1 \mathrm{c}$ foi associado à ISC, sendo que o risco de ISC aumentou à medida que o nível de HbA1cultrapassou 7,5 mg/dL (CANCIENNE JM, et al, 2017a; PENG W, et al., 2019). Entretanto, em outro estudo, o nível de HbA1c apontado como fator de risco para ISC foi $\geq 7 \mathrm{mg} / \mathrm{dL}$ (HIKATA T, et al., 2013). Na mesma vertente, Hwang Ju, et al. (2019), em sua pesquisa concluíram que o nível glicêmico $\geq 6,9 \%$ apontado por meio da $\mathrm{HbA} 1$ cfoi considerado fator de risco para ISC (HWANG JU, ET AL., 2019).Os diferentes valores apontados demonstram a falta de parâmetros para sustentar o manejo clínico adequado a fim de prevenir a ISC nos pacientes submetidos a cirurgias.

Em três pesquisas retrospectivas os resultados identificaram, entre outros fatores, que o DM está relacionado ao aumento do risco para ISC (PENNINGTON Z, et al., 2019; SATAKE K, et al., 2013; HIKATA T, et al, 2013). De acordo com os resultados encontrados no estudo de Pennington Z, et al. (2019), os pacientes do grupo de infecção apresentavam maior prevalência de DM $(48,5 \%)$ além de IMC maior e maior tempo de internação.

O DM, entre outros fatores, foi apontado como preditor independente significativo $(O R=2,12)$ para ISC o que sugere que o controle rigoroso dos níveis glicêmicos no período perioperatório, para pacientes com DM (de alto risco), porém de forma cautelosa, principalmente em idosos devido ao risco de hipoglicemia com consequente queda e fratura, pode diminuir o risco de ISC. Nesse mesmo estudo de Pennington Z, et al. (2019), evidenciou-se que a cada $10 \mathrm{mg} / \mathrm{dL}$ de glicemia média reduzida no primeiro dia pós-operatório, diminuiria em $25 \%$ a probabilidade de ISC. Na mesma vertente dos resultados encontrados no artigo de Pennington Z, et al. (2019), em coorte retrospectiva desenvolvida por Satake K, et al. (2013), envolvendo 566 pacientes com e sem DM os resultados mostraram que a incidência de ISC foi maior em aproximadamente nove vezes nos pacientes com DM quando comparados aos pacientes sem DM, no entanto, o nível glicêmico ideal para reduzir o risco de ISC não foi apontado.

Em estudo retrospectivo com 345 pacientes, com e sem a condição de DM, submetidos à cirurgia de coluna lombar e vertebral os resultados evidenciaram que o nível glicêmico no período pré-operatório foi significativamente maior nos pacientes com ISC quando comparados aos pacientes sem esse tipo de infecção. Foi demonstrado nos resultados que os pacientes com DM e que apresentavam valor médio da HbA1c de 6,6\% (variação de 6,1-6,9\%) não desenvolveram ISC, no entanto, os pacientes com DM não controlado que apresentaram HbA1c média de 7,4\% (variação de 7,0 a 8,1\%) apresentaram taxa de ISC de $35,3 \%$. Esses dados sugerem que a redução de ISC é influenciada pelo controle glicêmico em longo prazo e os resultados concluíram que um efetivo controle glicêmico por, pelo menos, 6 a 12 semanas antes da cirurgia, foi associado a um menor risco de ISC em pacientes com DM (HIKATA T, et al., 2013).

Resultados semelhantes aos resultados supra citados foram encontrados em dois estudos em que os autores conseguiram avaliar que pacientes com DM (controlados e não controlados) têm maiores chances de ISC quando comparados aos pacientes sem DM, contudo, também não conseguiram estabelecer parâmetros quanto aos níveis glicêmicos que aumentam o risco de ISC; no primeiro estudo os autores utilizaram uma 
amostra de 2.568.994 pacientes submetidos a cirurgia de coluna lombar e, no segundo, a amostra foi composta por 1.602.145 pacientes submetidos a cirurgia degenerativa de coluna cervical. Ambos os estudos separam as amostras em três grupos, sendo eles: DM controlado, DM não controlado e sem DM. Os resultados do primeiro estudo evidenciaram que os pacientes com DM (controlados e não controlados) tiveram maiores chances de apresentarem ISC quando comparados aos pacientes sem DM (OR de 1,36 e 2,61 respectivamente), resultados semelhantes foram evidenciados no segundo estudo quando os resultados demonstraram que pacientes com DM (controlados e não controlados) tiveram maiores chances de ISC ao serem confrontados com pacientes sem DM, obtendo um OR de 1,91 e 4,9 respectivamente (GUZMAN JZ, et al., 2014; GUZMAN JZ, et al., 2014).

Conforme mencionado no início dessa discussão, a hiperglicemia perioperatória pode ser prejudicial, independentemente da condição de DM, dados que vão ao encontro do estudo desenvolvido por Bellusse GC, et al. (2020), o qual envolveu 284 pacientes submetidos a cirurgias eletivas abdominais, quando os resultados apontam a hiperglicemia como fator de risco independente para ISC quando avaliadas ao final da cirurgia e 12 horas após. Nesse estudo os resultados evidenciaram que, quanto à gravidade da hiperglicemia, foi identificado efeito dose-resposta, ou seja, o risco de ISC aumentava à medida que a exposição a hiperglicemia aumentava, no entanto, não foram estabelecidos pontos de corte quanto aos níveis glicêmicos.

Outra informação relevante encontrada nos resultados desse estudo foi que a fração atribuível apontou que se a condição de hiperglicemia tivesse sido evitada a ISC não ocorreria em mais de $60 \%$ dos casos, dados que vão ao encontro de outros estudos que apontam que cerca de $40 \%$ a $60 \%$ das ISC podem ser evitadas e a prevenção torna-se fundamental na prática dos profissionais de saúde (SPRUCE L, 2014). Esses resultados sugerem a necessidade de implementação de protocolo no período perioperatório no intuito de auxiliar os profissionais de saúde envolvidos quanto ao manejo adequado dos níveis glicêmicos com vistas a redução da ISC.

Estudo de caso controle retrospectivo, incluído nessa R.I., analisou 5.194 pacientes submetidos à cirurgia de coluna lombar, cujo objetivo foi avaliar a associação do controle glicêmico perioperatório em pacientes com diabetes submetidos à descompressão lombar com a incidência de ISC profunda, e calcular um limite de $\mathrm{HbA1c}$ que pudesse relacionar-se com ISC. Os resultados conseguiram determinar, utilizando como ferramenta uma curva que relacionava o intervalo de hemoglobina glicada com a ocorrência de ISC, que o nível de HbA1c $\geq 7,5$ aumentava o risco de ISC (CANCIENNE JM, et al.,2017).

Resultado semelhante foi descrito em coorte retrospectiva que avaliou 3.341 pacientes que realizaram cirurgia de coluna cervical com objetivo de avaliar associação entre níveis de HbA1c perioperatórios em pacientes com DM e a incidência de ISC profunda e, como objetivo secundário, identificar um limiar de HbA1c acima do qual o risco de ISC aumenta progressivamente em pacientes com DM. O estudo conseguiu relacionar o aumento da ocorrência de ISC quando o nível de $\mathrm{HbA} 1 \mathrm{c} \geq 7,5$, tal resultado foi obtido através do ponto de inflexão de uma curva que relacionava o intervalo de $\mathrm{HbA1c}$ com a ocorrência de ISC, método semelhante ao utilizado no estudo anterior (CANCIENNE JM, et al., 2017).

Estudo de coorte retrospectiva que envolveu 523 pacientes com DM submetidos à cirurgia lombar e teve como objetivo analisar os fatores de risco para ISC nessa população, os resultados evidenciaram a ocorrência de ISC em $7 \%$ dos pacientes analisados, sendo maior em pacientes com $\mathrm{HbA} 1 \mathrm{c} \geq 7,5$ quando comparados aos pacientes com nível de HbA1c $<7,5$ (OR = 4,62) (PENG W, et al.,2019). Em coorte retrospectiva de Huwang JU, et al. (2019), a análise incluiu 92 pacientes submetidos à cirurgia de coluna lombar e teve como objetivo relacionar o nível de $\mathrm{HbA} 1 \mathrm{c}$ com incidência de ISC. Os resultados evidenciaram que o nível de $\mathrm{HbA} 1 \mathrm{c}$ no pré-operatório foi maior nos pacientes com ISC do que nos pacientes sem ISC, e demonstrou que pacientes com $\mathrm{HbA} 1 \mathrm{c} \geq 6,9 \%$ apresentavam maiores chances de ISC quando comparados aos pacientes com $\mathrm{HbA} 1 \mathrm{c}<6,9 \%(\mathrm{OR}=4,5)$.

Essa divergência entre os estudos quanto aos níveis glicêmicos sugere a falta de consenso na literatura sobre o nível ideal no período perioperatório. A Agência Nacional de Vigilância Sanitária preconiza níveis glicêmicos no pré-operatório e no pós-operatório imediato $<180 \mathrm{mg} / \mathrm{dl}$ para pacientes criticamente doentes 
no entanto, recomendação recente do Centers for Disease Control na Prevention (CDC) preconiza uma glicemia perioperatória $\leq 200 \mathrm{mg} / \mathrm{dl}$ (AGÊNCIA NACIONAL DE VIGILÂNCIA SANITÁRIA, 2017; CENTERS FOR DISEASE CONTROL AND PREVENTION, 2017).

Barker $\mathrm{P}$, et al. (2015), recomendam a dosagem dos níveis glicêmicos (utilizando-se a glicemia capilar devido ao fácil acesso) na admissão do paciente, antes da indução anestésica e o monitoramento regular durante o procedimento cirúrgico (de hora em hora ou com intervalos menores em caso de resultados alterados).

Nota-se que escassez de evidências quanto ao manejo perioperatório das medicações devido à falta de concordância entre os diferentes guidelines e, dessa maneira, julga-se necessário maior número de ensaios clínicos a fim de determinar o planejamento adequado para o tratamento desses pacientes (PONTES JPJ, et al., 2018).

\section{CONSIDERAÇÕES FINAIS}

Os artigos mostraram que a hiperglicemia perioperatória, independentemente da condição de DM, está relacionada a ocorrência de ISC. Sendo assim, torna-se evidente a necessidade de implementação de medidas que garantam o monitoramento dos níveis glicêmicos dos pacientes que serão submetidos a procedimentos cirúrgicos, independentemente da condição de DM. No entanto, observou-se a falta de parâmetros quanto ao nível glicêmico ideal, o que aponta a necessidade de novos estudos capazes de nortear essa lacuna.

\section{REFERÊNCIAS}

1. AGÊNCIA NACIONAL DE VIGILÂNCIA SANITÁRIA. Critérios Diagnósticos das Infecções Relacionadas à Assistência à Saúde: nota técnica. Brasília, 2019.

2. AGÊNCIA NACIONAL DE VIGILÂNCIA SANITÁRIA. Medidas de Prevenção de Infecção Relacionada à Assistência à Saúde. Brasília, 2017.

3. BARKER P, et al. Perio-operative management of the surgical patient with diabetes 2015: association of anaesthetists of Great Britain and Ireland. Anaesthesia, 2015; 70:1427-40.

4. BELLUSSE GC, et al. Effect of perioperative hyperglycemia on surgical site infection in abdominal surgery: A prospective cohort study. American Journal of Infection Control, 2020; 40:781-85.

5. BEZERRA WR, et al. Ocorrência de incidentes em um centro cirúrgico: estudo documental. Revista Eletrônica de Enfermagem, 2015; 17(4): 24-27.

6. BRASIL. Ministério da Saúde. Portaria № 2616, de 12 maio de 1998. Expede na forma de anexos, diretrizes e normas para a prevenção e controle das infecções hospitalares. Diário Oficial da União, 1998.

7. CANCIENNE JM, et al. Perioperative hemoglobin A1C as a predictor of deep infection following single level lumbar decompression in patients with diabetes. The Spine Journal, 2017; 17(8): 1100-1105.

8. CANCIENNE JM, et al. The Association of Perioperative Glycemic Control with Deep Postoperative Infection After Anterior Cervical Discectomy and Fusion in Patients with Diabetes. World Neurosurgery, 2017; 102: 13-17.

9. CARVALHO RLR, et al. Incidence and risk factors for surgical site infection in general surgeries. Rev. Latino-Americana de Enfermagem, 2017; 25: 2848.

10. CENTERS FOR DISEASE CONTROL AND PREVENTION. Surgical site infection (SSI) event. Procedure-associated Module SSI. Atlanta: Centers for Disease Control and Prevention, 2017.31p.

11. DAVIS MC, et al. Preoperative hyperglycemia and complication risk following neurosurgical intervention: a study of 918 consecutive cases. Surgical Neurology International, 2012; 3(49):13-20.

12. ERCOLE FF, et al. Revisão integrativa versus revisão sistemática. Revista Mineira de Enfermagem, 2014; 18(1): 912.

13. FRISCHA., et. al. Prevalence and clinical outcome of hyperglycemia in the perioperative period in noncardiac surgery. Diabetes Care,2010; 33(8):1783-88.

14. FUSCO SFB, et al. Infecção de sítio cirúrgico e seus fatores de risco em cirurgias de cólon. Revista da Escola de Enfermagem da USP, 2016; 50(1): 43-49.

15. GOMES, JAP, et al. Instrumentos para avaliar a qualidade e segurança no bloco operatório: revisão integrativa. Cogitare Enferm, 2016; 21: 1-9.

16. GUEDES AA. A importância do controle glicêmico perioperatório. Revista Médica de Minas Gerais, $2010 ; 20$ (4 Supl 1): 3-6.

17. GUZMAN JZ, et al. Outcomes and complications of diabetes Mellitus on patients undergoing degenerative lumbar spine surgery. Spine, 2014; 39(19): 1596-1604. 
18. GUZMAN JZ, et al. The impact of diabetes mellitus on patients undergoing degenerative cervical spine surgery. Spine, 2014; 33(20): 1656-65.

19. HIKATA T, et al. High preoperative hemoglobin A1c is a risk factor for surgical site infection after posterior thoracic and lumbar spinal instrumentation surgery. Journal of Orthopaedic Science, 2013; 19(2): 223-28.

20. HWANG JU, et al. Importance of hemoglobin A1c levels for the detection of post-surgical infection following singlelevel lumbar posterior fusion in patients with diabetes. Korean Journal of Neurotrauma, 2019; 15(2): 150-58.

21. KUMAR V, et al. Robbins, patologia básica. 9 ed. Rio de Janeiro: Elsevier, 2013; 31-39 p.

22. MENDES KDS, et al. Revisão integrativa: método de pesquisa para a incorporação de evidências na saúde e na enfermagem. Texto contexto - enfermagem, 2008; 17(4): 758-64.

23. PALERMO NE, et al. Stress hyperglycemia during surgery and anesthesia: pathogenesis and clinical implications. Current Diabetes Reports,2016; 16(3): 3339.

24. PENG W, et al. Multivariate analysis of incision infection after posterior lumbar surgery in diabetic patients: a singlecenter retrospective analysis. Medicine, 2019; 98(23): 1-7.

25. PENNINGTON Z, et al. Persistent postoperative hyperglycemia as a risk factor for operative treatment of deep wound infection after spine surgery. Neurosurgery. Publicado eletronicamente antes da versão impressa em: 2019 set. 25.

26. PEREIRA MS, et al. A infecção hospitalar e suas implicações para o cuidar da enfermagem. Texto e Contexto Enfermagem, 2005; 14 (2): 250-57.

27. PONTES JPJ, et al. Avaliação e manejo perioperatório de pacientes com diabete melito: um desafio para o anestesiologista. Revista Brasileira de Anestesiologia, 2018; 68 (1): 75-86

28. SANTOS CMC, et al. Estratégia PICO para a construção da pergunta de pesquisa e busca de evidência. Revista Latino-Americana de Enfermagem, 2007; 15(3):508-11.

29. SATAKE K, et al. Predisposing factors for surgical site infection of spinal instrumentation surgery for diabetes patients. European Spine Journal, 2013; 22(8): 1854-58.

30. SOBECC. Práticas recomendadas SOBECC -Centro de Material e Esterilização, Centro Cirúrgico, Recuperação PósAnestésica. 7ํㅡㄹ. ed. São Paulo: SOBECC Associação, 2017.

31. SOCIEDADE BRASILEIRA DE DIABETES. Diretrizes Sociedade Brasileira de Diabetes: 2019-2020. São Paulo, 2019.

32. SPRUCE L. Back to basics: preventing surgical site infections. AORN Journal,2014; 99(1):600-11.

33. THOMPSONMB, et al. Perioperative management of patients with diabetes and hyperglycemia undergoing elective surgery. Currents Diabetes Reports, 2016; 16(2):2-9.

34. TOWNSEND JRCM, et al. Tratado de Cirurgia: a base biológica da prática cirúrgica moderna. 18th ed. Rio de Janeiro: Elsevier, 2015; 147p. 